\title{
Functional Characterization of 9-/13-LOXs in Rice and Silencing Their Expressions to Improve Grain Qualities
}

\author{
Moytri RoyChowdhury, ${ }^{1}$ Xiaobai Li, ${ }^{2,3}$ Hangying Qi, ${ }^{4}$ Wenxu Li, ${ }^{5}$ \\ Jian Sun, ${ }^{2}$ Cheng Huang, ${ }^{4}$ and Dianxing $\mathrm{Wu}^{2}$ \\ ${ }^{1}$ Department of Horticulture, Washington State University, Pullman, WA 99164, USA \\ ${ }^{2}$ State Key Lab of Rice Biology, International Atomic Energy Agency Collaborating Center, Zhejiang University, \\ Hangzhou, Zhejiang 310029, China \\ ${ }^{3}$ Zhejiang Academy of Agricultural Sciences, Hangzhou, Zhejiang 310021, China \\ ${ }^{4}$ Agricultural Extension Extending Stations, Shaoxing \& Zhuji Agricultural Bureau, Shaoxing, Zhejiang 312000, China \\ ${ }^{5}$ Institute for Wheat Research, Henan Academy of Agricultural Sciences, Zhengzhou, Henan 450002, China
}

Correspondence should be addressed to Xiaobai Li; hufanfan1982815@gmail.com and Dianxing Wu; dxwu@zju.edu.cn

Received 5 December 2015; Accepted 24 April 2016

Academic Editor: Sudhir Sopory

Copyright (C) 2016 Moytri RoyChowdhury et al. This is an open access article distributed under the Creative Commons Attribution License, which permits unrestricted use, distribution, and reproduction in any medium, provided the original work is properly cited.

\begin{abstract}
Lipoxygenases (LOXs) are involved in oxidative rancidity and render rice unsuitable for human consumption. Here, RNA interference- (RNAi-) induced gene expression inhibition was used to analyze the functions of the bran/seed-specific LOXs in rice. $r$ - LOX1 and L-2 (9-LOX category) were the candidate genes expressing a bran/seed-specific LOX, while RCI- 1 was (13-LOX category) a plastid-specific LOX. Real-time PCR showed that three LOXs were cultivar/tissue specific expression on a certain level. $r 9-L O X 1$ and $L-2$ were generally much higher in active bran/seed than in stabilized bran, mature seed, and regenerated plant. $R C I-1$ was barely expressed in seed. In transgenic lines, $r 9-L O X 1$, as well as $L-2$, expression was dramatically downregulated, compared to the nontransgenic controls. SPME/GC-MS analysis of $r 9$-LOX1 RNAi transgenic lines showed 74.33\% decrease in nonanal content (formed during oxidation of linoleic acid by lipoxygenase), but $388.24 \%$ increase in acetic acid and $184.84 \%$ hexanal (direct products of 13-LOX). These results indicate that $r 9-L O X 1$ positively regulates the amount of nonanal but negatively regulates acetic acid and hexanal. The negative regulation may be due to a mechanism of negative feedback between LOX family members. The information will help comprehensively understand the function of the bran/seed-specific LOXs, $r 9$-LOX1, and improve the storage quality in the future.
\end{abstract}

\section{Introduction}

Lipoxygenases (LOXs) ubiquitously exist in the seeds of many plant species. Seed LOXs may be involved in the fatty acid peroxidation, lipids storage, production of growth regulators, responses to pathogens, and nitrogen storage [1]. The influence of LOXs on favor quality is reported in soybean and rice. Soybean LOXs are involved in the production of volatile compounds (such as n-hexanal) which are associated with grassy beany and rancid off-flavors in soybean and soy foods [2]. In stored rice seeds, LOX activities are also present [3], and hexanal is the predominant component of stale off-flavors derived from lipid peroxidation [4]. Rice seed LOXs are also involved in lipid peroxidation in rice seeds [5]. Since the absence of LOXs can alleviate seed lipid peroxidation, LOXs are always the targets of genetic modifying during molecular breeding. Soybean "IA2025," a LOX triple null-mutation soybean line, can be used to produce tofu and soymilk with improved flavor and sensory qualities [6-8]. In barley, a LOX-1 deficient line "DaikeiLM1" is gained from "Karl M2" through sodium azide treatment [9]. This mutated line can effectively improve flavor stability in beer [10]. In rice, 
TABLE 1: Primer sequences of three LOX genes for cloning.

\begin{tabular}{|c|c|c|c|}
\hline Gene (accession number) & Primers & Sequence & Product size \\
\hline \multirow{2}{*}{ r9-LOX (AB099850) } & CDS r9-LOX1F & CACCGCTGGACGAGAACCCAGAGAAG & \multirow{2}{*}{$238 \mathrm{bp}$} \\
\hline & CDS $r 9-L O X 1 \mathrm{R}$ & CAGGGGGTCCTTGTTCATGTT & \\
\hline \multirow{2}{*}{ RCI-1 (AJ270938) } & CDSRCI-1F & CACCCGAAGGCTACTTCAGGGAGGTG & \multirow{2}{*}{214 bp } \\
\hline & CDSRCI-1R & CGTGTCCCTGACAAGTTGGATG & \\
\hline \multirow{2}{*}{$L-2(\mathrm{X} 64396)$} & $\mathrm{CDS} L-2 \mathrm{~F}$ & CACСССТCGCСАТСТАСТАССССАACG & \multirow{2}{*}{$217 \mathrm{bp}$} \\
\hline & $\mathrm{CDSL}-2 \mathrm{R}$ & GTACGGGTACTGCCCGAAGTTG & \\
\hline
\end{tabular}

the absence of sLOX3 in the seeds of "DawDam" results in less stale flavor [11].

According to the differences of substrates at carbon 9 or 13 of the hydrocarbon backbone, LOXs are classified into 9LOXs and 13-LOXs [12]. Some plants may have predominant 9-LOX or 13-LOX activity, while some other plants may have equally 9-LOX and 13-LOX activities. According to the protein sequences, LOXs are classified into three types as described by Mizuno et al. [13]: Type I is localized in chloroplast and stress inducible; Type II is localized in cytoplasm, derived from dicots, and not stress inducible; Type III is localized in the cytoplasm, derived from monocots and related to seed germination. Type I LOXs have a transit peptide, lacking in Type II and Type III LOXs. Three isozymes in Type III LOXs (LOX1/L-1, LOX2/L-2, and LOX3/L-3) have been identified in Oryza sativa embryos. Among them, LOX3 is the most abundant one [14]. The catalytic LOX domains in both Type II and Type III LOXs have oxygen binding and oxylipin synthesis sites. The enzyme r9-LOX1 belongs to Type III and is the focus of this study. Three Type III LOXs ( $L-1, L-2$, and $L-3)$ in developing rice seeds have been identified [15]. L-2 (Type III) derives from 3-day-old seedlings [16], while $R L L$ (Type I) is isolated from rice leaves [17]. $L-3$ (Type III) is the major component of these isozymes and accounts for 80$90 \%$ of their total activities [18]. The lack of $L-3$ in DawDam leads to a decrease of lipid peroxidation and the alleviation of stale flavor accumulation by hexanal, as well as pentanal and pentanol compounds during storage [19]. Unfortunately, DawDam could not be used for rice production due to its poor agronomic characters [18]. It is speculated that the lack of LOX-3 may affect the metabolism in the whole plant.

In order to effectively improve rice grain storage qualities, inactivation of some lipase and/or LOXs should be achieved without compromising nutritional value and agronomic qualities. RNAi could be an ideal method to achieve this goal based on its applications on improving the nutritional quality of various crops [20]. Therefore, the objectives of this study were to select two candidate seed/bran LOXs gene(s), develop suitable constructs for stable RNAi in rice, and study the potential benefits of silencing bran/seed-specific LOXs.

\section{Materials and Methods}

2.1. Plant Materials. The japonica rice cultivar "LaGrue" was used to clone target genes and develop transgenic plants. The tissues from DawDam, Taipei-309, and transgenic lines were used for gene expression analysis.
2.2. Genomic DNA Extraction and PCR Analysis. Genomic DNA was extracted from the leaf tissues of Taipei-309 seedlings by Nucleon Phytopure Plant DNA Extraction kit (Amersham Biosciences) according to the manufacturers' protocol.

Two Type III LOXs, r9-LOX1 (accession number: AB099850) and L-2 (accession number: X64396), and a leafspecific LOX RCI-1 (accession number: AJ270938), as well as RCI-1 (accession number: AJ270938) were selected for study. Interestingly, the L-3 (accession number: E03480) sequence was noted to be identical to the L-2 sequence (accession number: X64396) known to be distributed in rice embryo and have C9 specific LOX activity [13]. The nucleotide sequences of $L-2$ and $L-3$ (Type III) were identical. The primers for these LOX genes were listed in Table 1. Gene sequences for inverted repeats were amplified using thermostable, proofreading DNA polymerase from Stratagene (La Jolla, CA, USA). The CACC sequence was added to the $5^{\prime}$ end of forward primer for facilitating directional incorporation into Invitrogen's pENTR/D-TOPO entry vector. PCR was performed by using KOD-Plus-Neo (TOYOBO, Japan) and then the products were separated by $0.7 \%$ agarose gel. The target fragments were collected using Qiangen gel purification kit.

2.3. Construction of $h p R N A i$ Vectors. RNAi constructs were prepared following the Gateway System protocol [21]. For constructing hpRNAi vectors, the target gene fragments were firstly cloned into TOPO pENTR following the Gateway System protocol (Invitrogen, Carlsbad, CA). The transformation of Agrobacterium tumefaciens EHA105 with the pANDA vector was performed using the Freeze and Thaw method [22]. Agrobacterium-mediated transformation in rice was carried out as described previously $[23,24]$, with minor modifications [25]. The positive control EHA105 was transformed with pWHNG vector.

2.4. Rice Transformation. The transformation of Agrobacterium tumefaciens EHA105 with the pANDA vector was performed using the Freeze and Thaw method [22]. Agrobacterium-mediated transformation in rice was carried out as described previously $[23,24]$, with minor modifications [25]. The positive control EHA105 was transformed with pWHNG vector.

Transformation was carried out using 21-day-old calli grown on B5 medium with $2.2 \mathrm{mg} / \mathrm{L} \mathrm{2,4} \mathrm{D.} \mathrm{After} \mathrm{being}$ treated with liquid cocultivation medium (YEP containing EHA105 at $600 \mathrm{OD}$ of 1) for $20 \mathrm{~min}$, the calli were transferred to a solid cocultivation medium (B5 medium with $2.2 \mathrm{mg} / \mathrm{L}$ 
TABLE 2: Rate of Agrobacterium-mediated transformation.

\begin{tabular}{|c|c|c|c|c|c|}
\hline Constructs & Total explants & Transformed plants & $\begin{array}{c}\text { Regeneration } \\
\text { percentage (\%) }\end{array}$ & Confirmed transformation & $\begin{array}{c}\text { Transformed plants } \\
\text { percentage (\%) }\end{array}$ \\
\hline Negative control & 7 & 4 & 57.0 & & \\
\hline EHA105 + pWHNG & 40 & 4 & 10.0 & 2 & 5.0 \\
\hline EHA105 + r9-LOX & 160 & 12 & 7.5 & 4 & 2.0 \\
\hline EHA105 + L2 & 200 & 22 & 11.0 & 7 & 3.0 \\
\hline EHA105 + RCI-1 & 180 & 12 & 6.6 & 5 & 3.0 \\
\hline Mean & & & $8.78 \%$ & & $3.25 \%$ \\
\hline
\end{tabular}

2, $4 \mathrm{D}$ and 100 um acetosyringone) for 3 days. After cocultivation, the transformed calli were cultured in solid B5 medium with $2.2 \mathrm{mg} / \mathrm{L} \mathrm{2,} 4 \mathrm{D}$ and $250 \mathrm{mg} / \mathrm{L}$ Claforan for 7 days and then transferred to the selective medium (B5 medium with $2.2 \mathrm{mg} / \mathrm{L} \mathrm{2}, 4 \mathrm{D}, 250 \mathrm{mg} / \mathrm{L}$ Claforan, and $25 \mathrm{mg} / \mathrm{L}$ geneticin) for 15 days. The proliferated, yellowish white calli were transferred to the same medium for another 15 days. Resistant calli were transferred to regeneration medium (B5 medium with $0.03 \mathrm{mg} / \mathrm{L}$ Picloram and $0.35 \mathrm{mg} / \mathrm{L} \mathrm{BA}$ ) for 15 days. After 15 days, the medium was replaced with fresh medium for another 15 days. Seedlings were then transferred to fresh B5 medium without antibiotics for 15 days and then transferred to the planters in greenhouse.

2.5. Real-Time PCR. Total RNA was extracted from mature seed, 2-day-old germinating seed, imbibed seed, and stabilized bran and 35-day-old regeneration plants of different varieties rice or transgenic plants using Trizol (Invitrogen, Carlsbad, CA) [26] and treated with RNase-free DNase I (TaKaRa, Shuzo, Kyoto, Japan) to remove potential contaminating genomic DNA. First Strand cDNA was synthesized using SuperScript TM II RT kit (Invitrogen, Carlsbad, CA).

All samples were amplified in triplicate from the same RNA preparation and the mean values were calculated. The primers for real-time analysis were designed based on gene specific sequences (see Supplementary Table S1 in Supplementary Material available online at http://dx.doi.org/10.1155/ 2016/4275904). RT-PCR was performed by using Syber Green $^{\circledast}$ (Roche Diagnostics) on $\mathrm{C} 1000^{\mathrm{T}}$ Thermal Cycler equipped with CFX96 ${ }^{\mathrm{TM}}$ Real-Time System (Bio-Rad, USA). Primer specificity was tested by isolated PCR products in high resolution gel electrophoresis. Melting curve program (60$95^{\circ} \mathrm{C}$ ) was performed with the heating rate at $0.10^{\circ} \mathrm{C} / \mathrm{sec}$, continuous fluorescence measurement, and a final cooling step to $40^{\circ} \mathrm{C}$.

To determine relative amount of LOXs mRNA transcript in different rice tissues, we applied the REST (Relative Expression Software Tool) calculations based on the Ct (cycle threshold) values at a constant fluorescence level [27, 28]. The relative expression/mRNA transcript ratio of each $L O X$ was computed based on its RT-PCR efficiency and the crossing point difference between LOX and Actin control.

2.6. SPME GC/MS Analysis. Samples for SPME GC/MS were prepared by first hulling the seeds using hands or table top rice huller depending on the amount of rice and the easiness to hull the seeds. The hulled seeds were then milled to $10 \%$ (removal of bran layer) degree using "WonderHand Mill" [29]. The native and transgenic bran was subjected to identical storage conditions to maintain identical moisture level. SPME GC/MS analysis was performed using a method modified by Grimm et al. [30].

\section{Results}

3.1. RNAi Vector Construct and Development of Transgenic Plants. PCR results showed the correct sizes of LOX fragments with the range of 200-300 bp for 9r-LOX1 and $L-2$ (Supplementary Figure S1). The fragments of LOX genes were targets for RNAi shown as Figure 1. These fragments were initially linked to TOPO pENTR vector and then transferred into pANDA for hpRNAi expression (Supplementary Figure S2). The insertions of target fragments in pENTER vector and in pANDA were confirmed by PCR and restriction enzyme digestion (Supplementary Figures S3 and S4). Sequencing results revealed that all the sequences for $r 9-L O X 1, L-2$, and $R C I-1$ were consistent with the original sequences in the database only with a few mismatches. Moreover, the binary pWHNG vector containing GUS was chosen as positive control of Agrobacterium-mediated transformation (Supplementary Figure S5). The hpRNAi vector and pWHNG were transformed into rice by Agrobacterium-mediated transformation of rice mature embryo. A total of 160 to 200 explants were used for $r$-LOX1, LOX2, or RCI-1 gene silencing plants and 40 explants were for generation of "positive control" transgenic plants. After selection and regeneration, 12 to 22 transformed $\mathrm{T}_{0}$ plants were produced for hpRNAi vectors and four for pWHNG vector, with average regeneration rate of $8.78 \%$. The transgenic plants were further confirmed by PCR using vector specific primers (Supplementary Figure 6 and Table 2). The result showed that two to seven PCR $\mathrm{T}_{0}$ plants were positive for the vectors, with average rate of positive confirmation of $3.25 \%$. The plants were grown in greenhouse until mature, and then $\mathrm{T}_{1}$ seeds were harvested and grown for two generations to obtain stable transgenic plants. The different tissues were collected for the expression analysis of target gene. Schematic diagram for the transformation process is depicted in Figure 2.

3.2. Expression Pattern of LOXs. This study analyzed the expression patterns of two bran/seed-specific genes, $r 9$ $L O X 1$ and $L-2$, and one chloroplast-specific gene, $R C I-1$ 

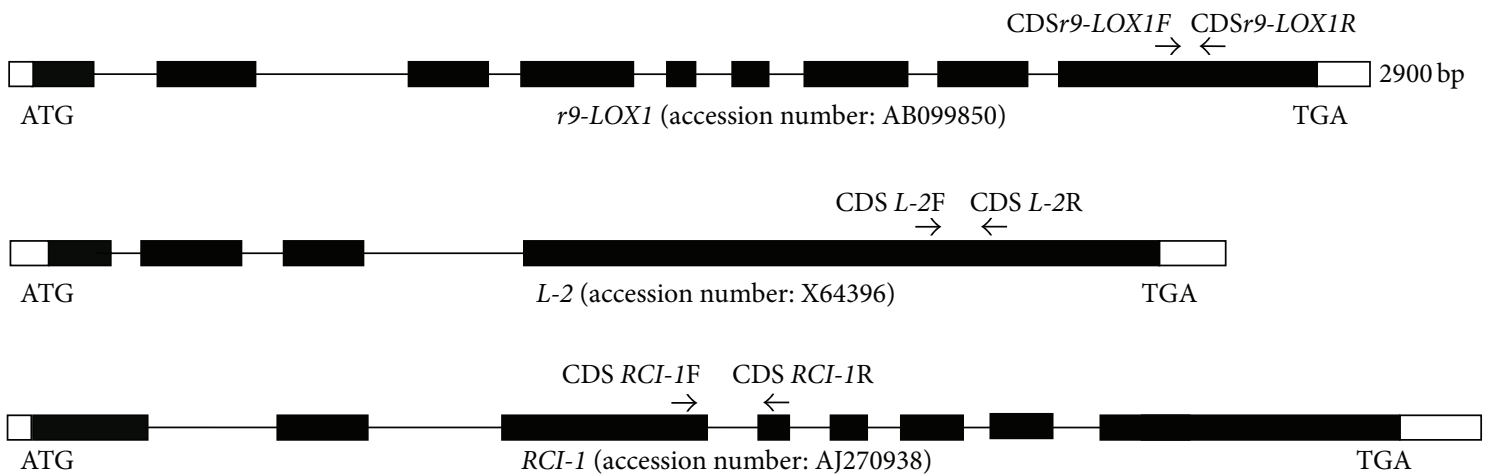

FIGURE 1: Gene structure and the target site for RNA interference.

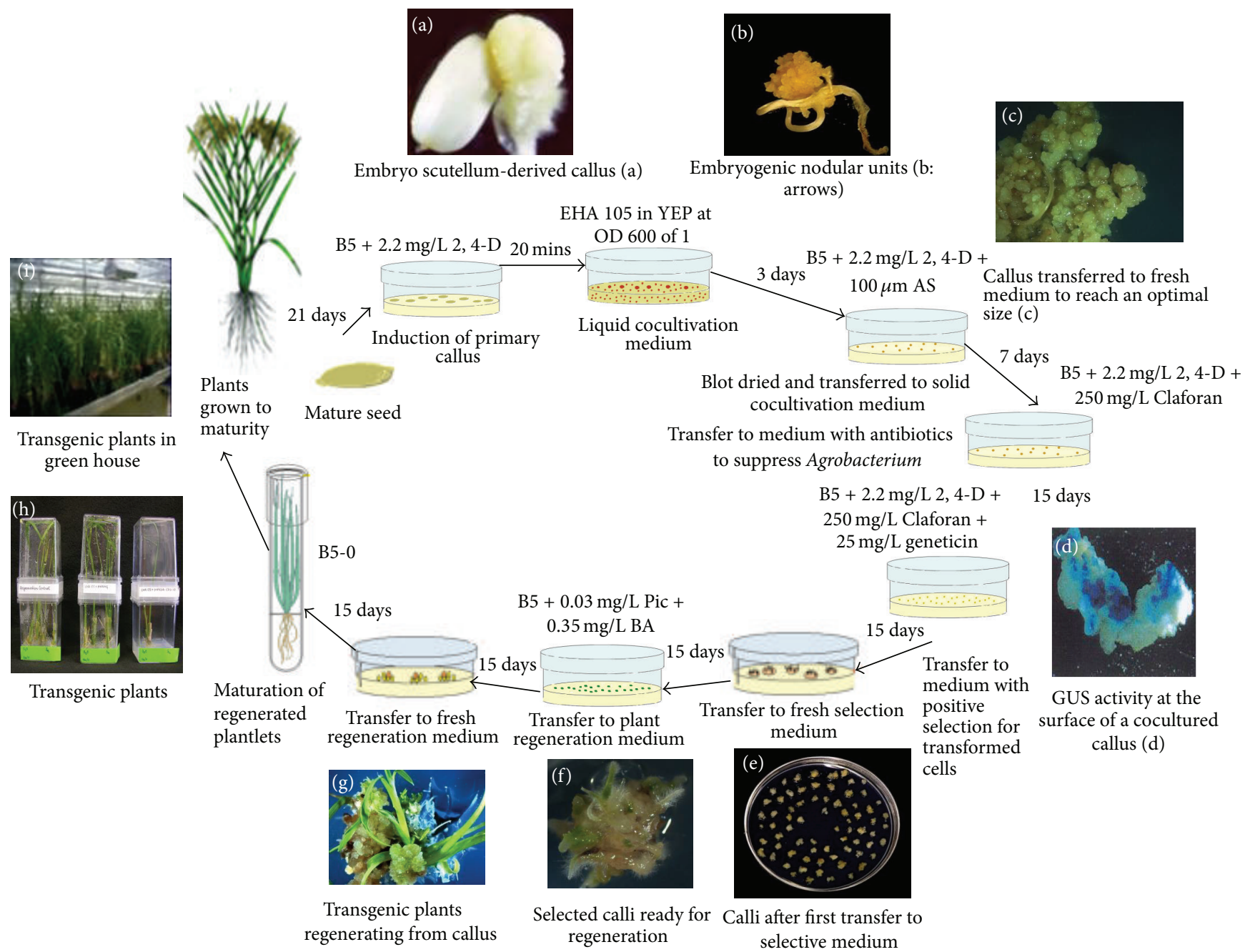

FIgURE 2: Agrobacterium-mediated transformation. The figure is a schematic diagram of the events involved in rice transformation.

(Figures 3(a), 3(b), and 3(c)). r9-LOX1 was highly expressed in germinating and imbibed seed, as well as Spring and Taipei bran. $L-2$ was also highly expressed both in germinating and imbibed seed, as well as Taipei bran. In contrast, $r 9-L O X 1$ and $L-2$ were both barely expressed in transgenic tissues. $R C I-1$ was highly expressed in "null" mutant "DawDam" imbibed seed, as well as transgenic tissue.

The expressions of these three LOXs showed tissue/ cultivar-specificity on a certain level (Figures 3(a), 3(b), and 3(c)). r9-LOX1 expression was generally much higher in active 


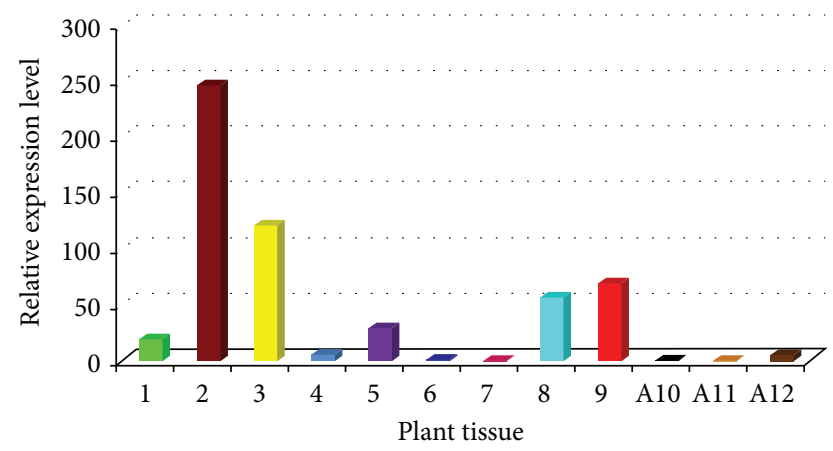
(1) Mature seed
(2) Germinating seed
(3) Imbibed seed
(4) Stabilized bran
(5) DawDam imbibed seed
(6) Regenerated plant
(9) Taipei bran
(A10) Transgenic $r 9-L O X 1$ callus
(A11) Transgenic $r$ 9-LOX1 plant
(A12) Transgenic $r 9-L O X 1$ seed

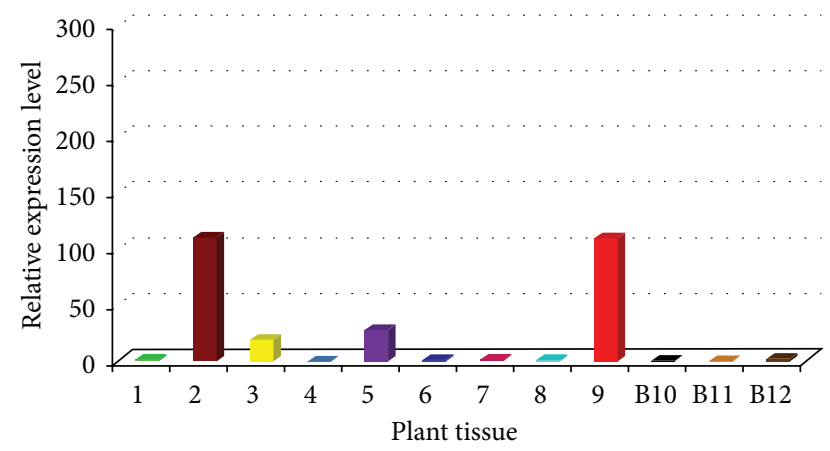
(1) Mature seed
(2) Germinating seed
(3) Imbibed seed
(7) DawDran bran
(4) Stabilized bran
(8) Spring bran
(5) DawDam imbibed seed
(6) Regenerated plant
Taipei bran
(B10) Transgenic $L-2$ callus
(B11) Transgenic $L-2$ plant
(B12) Transgenic $L-2$ seed

(a)

(b)

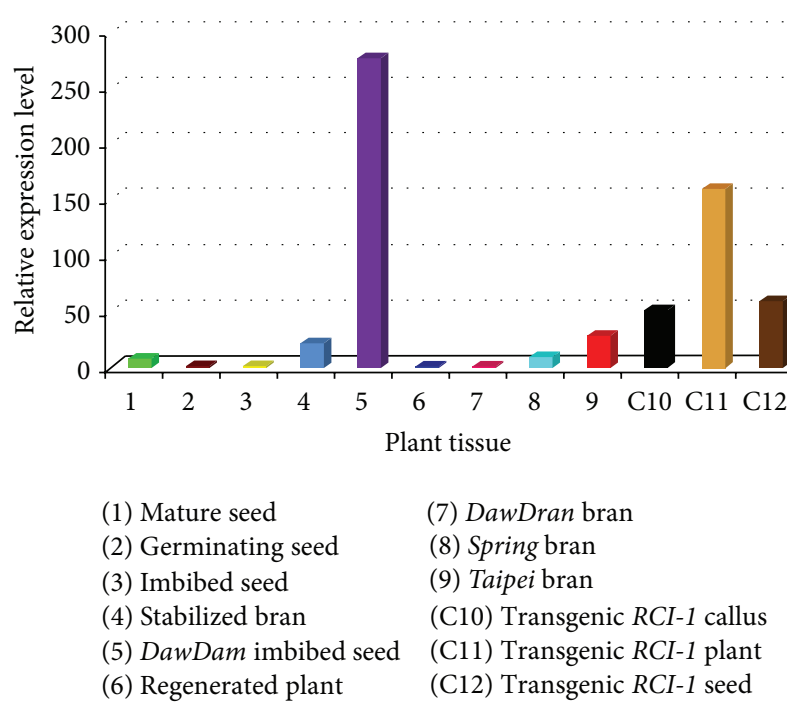

(c)

FIGURE 3: Relative expressions of three $L O X$ genes (a) $r 9-L O X 1$, (b) $L-2$, and (c) $R C I-1$. among transgenic and nontransgenic rice tissues.

bran/seed than in stabilized bran, mature seed, and regenerated plant. Among active bran/seed tissue, germinating seeds had the highest expression of $r 9-L O X 1$, followed by imbibed seed, Taipei bran, and Spring bran. Similarly, $L-2$ had relative high expression in active bran/seed tissue. Interestingly, the chloroplast-specific RCI-1 showed exceedingly high expression in the imbibed seeds of the null mutants "DawDam" and in all of the transgenic calluses, plants, and seeds (Figure 3(c)).

3.3. The Volatile Byproducts in r9-LOX1 RNAi Rice Bran. The volatile byproducts during lipid oxidation in nontransgenic and transgenic RNAi rice bran were examined by SPME/GCMS. Acetic acid and hexanal are the direct products of 13lipoxygenase, while nonanal is formed during the oxidation of linoleic acid by LOX. In SPME/GC-MS analysis, silencing r9-LOX1 coding region was obviously associated with the changes of byproducts during lipid oxidation. The contents of acetic acid were much higher in transgenic bran than in the control lines by $388.24 \%$, as well as hexanal by $184.84 \%$. In contrast, the contents of nonanal were significantly decreased in transgenic lines by $74.33 \%$ (Figure 4 ).

\section{Discussion}

4.1. RNAi Approach for Nutrition Improvement and Genetic Study in Crops. HGS-hpRNAi has been used to improve nutritional qualities in several crops. For example, it is used to successfully reduce $\gamma$-Gliadins in bread wheat [31]. Besides, it is used to reduce the expression levels of two starchbranching enzymes, SBEIIa and SBEIIb, which results in increased amylose content [32]. In transgenic peanut, knocking down oleate desaturase (FAD2) by RNAi can increase oleic acid content by $70 \%$ [33]. Moreover, the application of 


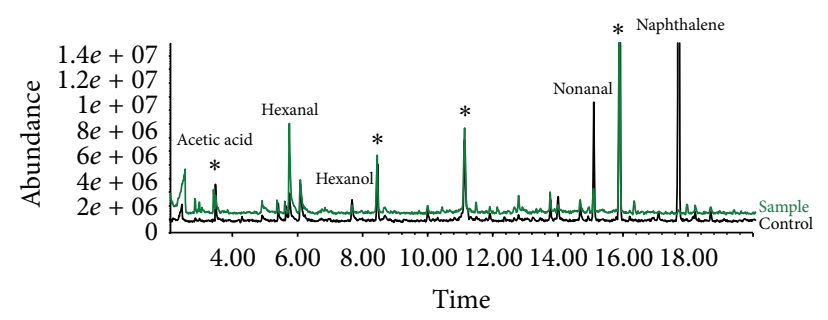

${ }^{*}$ A SPME contaminant

\begin{tabular}{lccc}
\hline Products in pathway & GC RT (mins) & Control area counts & Transgenic area counts \\
\hline Acetic acid & 2.44 & $6,866,451$ & $33,524,756$ \\
Hexanal & 5.75 & $9,019,178$ & $25,689,932$ \\
Nonanal & 15.11 & $22,363,505$ & $5,738,826$ \\
Naphthalene & 17.73 & $117,817,908$ & 0 \\
\hline
\end{tabular}

Note: $r$-LOX1 RNAi lines showed $74.33 \%$ decrease in nonanal, but $388.24 \%$ increase in acetic acid and $184.84 \%$ hexanal.

FIGURE 4: SPME/GC-MS analysis of the volatile byproducts by lipid oxidation in nontransgenic and transgenic rice bran of $r 9-L O X 1$.

this technology in our research could provide more insights into the functional characterization of rancidity-associated LOXs. Traditionally, studies of gene functions mainly relied on traditional breeding strategies which are time consuming. In the recent decade, RNAi have largely expedited this process. In this study, pANDA vector was adopted, since it is an efficient RNAi vector for rice and has been used for Agrobacterium transformation in rice over the years [21]. Our results showed that two bran/seed-specific LOXs' expression was suppressed in more than $90 \%$ of the transgenic plants, indicating the high efficiency of RNAi system.

4.2. LOXs' Expression Patterns. The expression of two bran/ seed-specific $L O X$ s and one plastid-specific $L O X$ were studied among different tissues. Two bran/seed-specific genes, $r 9$ $L O X 1$ and $L-2$, were both highly expressed in active seed including imbibed and germinating seed but barely expressed in stabilized bran, mature seed. In previous studies, it is reported that $r 9-L O X 1$ is expressed in rice imbibed seeds [13] and in tea plant seeds [34]. The similar expression pattern of the two bran/seed-specific LOXs may be due to their similar biological functions in rice bran/seeds and $71.3 \%$ homology [13]. Compared to $r 9-L O X 1, L-2$ showed about $15 \%$ decreased expression in germinating and imbibed seeds and less expression in Spring bran. The results are consistent with the previous study where an expressional variation is observed between the predicted bran/seed-specific LOXs in soybean [35]. The different expression patterns of LOX isozymes indicate that they may also play other different roles during seed developmental stages.

The expression patterns of $r 9-L O X 1$ and $L-2$ were significantly different from that of RCI-1 (Figures 3(a), 3(b), and 3(c)). The expressions of the two bran/seed-specific LOXs were low in null-mutation imbibed seed and rare in the transgenic $\mathrm{T}_{1}$ seeds with corresponding RNAi construct (Figures 3(a), 3(b), and 3(c)). Interestingly, RCI-1 is previously reported to be chloroplast-specific [36]. The chloroplastspecific RCI-1 showed an exceedingly high expression in the imbibed seeds of the null mutants as well as an expression at a certain level in Taipei bran (Figure 3(c)), which indicates that we should take $R C I-1$ as plastid-specific rather than the previously reported leaf-specific. In addition, $R C I-1$ expression was also observed in the seeds of transgenic lines (Figure 3(c)). Compared to $r 9-L O X 1$ and $L-2, R C I-1$ showed a high expression level in the imbibed seeds of null-mutation line DawDam, which might be caused by a mechanism of negative feedback between Type III LOX members in seeds.

4.3. The Biologic Functions of r9-LOX1. In SPME/GC-MS analysis, $r 9-L O X 1$ RNAi line showed $74.33 \%$ reduction of nonanal (formed during the oxidation of linoleic acid by LOX). These results indicate that $r 9-L O X 1$ positively regulates the amount of nonanal. Previous study also discovers a positive correlation between nonanal concentration and LOX activity [37]. However, r9-LOX1 RNAi lines showed 388.24\% increase in the amount of acetic acid and $184.84 \%$ increase in hexanal (direct products of 13-LOX) (Figure 3), which may be due to a negative feedback mechanism between $L O X$ family members. Similarly, RCI-1 RNAi line did not exhibit efficient silencing effect, which may be due to the shared oxylipin synthesis domain between Type III and Type II LOXs [38]. These results suggest that the LOXs derived from different categories may coordinate with each other and play important roles in many biological processes. For example, the complete knockdown of Type III LOXs results in poor defense function and therefore leads to poor agronomic traits in DawDam.

\section{Conclusion}

The results suggest that $r 9-L O X 1$ plays roles in synthesis pathways of nonanal, acetic acid, and hexanal in rice bran/seed. Notably, it is very hard to reduce all of $L O X$ rancid byproducts by silencing $L O X s$, because of the complementary action of different members of LOX family. Since a number of LOXs also play crucial parts in some basic biological processes, silencing all of them in rice will result in plants with poor 
agronomic characters, like DawDam. The information will help understand the function of LOXs and improve the storage quality in the future.

\section{Competing Interests}

The authors declare that they have no competing interests.

\section{Authors' Contributions}

Moytri RoyChowdhury, Xiaobai Li, and Hangying Qi equally contributed to this paper.

\section{Acknowledgments}

The authors would like to thank Dr. Pace Lubinsky, USDAFAS, and Junli Zhang, University of California-Davis, for critical review of the paper, USDA, Dale Bumpers National Rice Research Center for providing the rice seeds, and Dr. Casey Grimm USDA-ARS for the assistance with GC/MS experiments. The study was supported by Science Technology Department of Zhejiang Province (0406) and the Major State Basic Research Development Program of China (2013CBA01403).

\section{References}

[1] J. Loiseau, B. L. Vu, M.-H. Macherel, and Y. Le Deunff, "Seed lipoxygenases: occurrence and functions," Seed Science Research, vol. 11, no. 3, pp. 199-211, 2001.

[2] D. S. Robinson, Z. Wu, C. Domoney, and R. Casey, "Lipoxygenases and the quality of foods," Food Chemistry, vol. 54, no. 1, pp. 33-43, 1995.

[3] B. S. Shastry and M. R. R. Rao, "Studies on lipoxygenase from rice bran," Cereal Chemistry, vol. 52, pp. 597-603, 1975.

[4] S. Chikubu, "Stale flavor of stored rice," Jarq-Japan Agricultural Research Quarterly, vol. 5, pp. 63-68, 1970.

[5] Q. Long, W. Zhang, P. Wang et al., "Molecular genetic characterization of rice seed lipoxygenase 3 and assessment of its effects on seed longevity," Journal of Plant Biology, vol. 56, no. 4, pp. 232-242, 2013.

[6] A. V. Torres-Penaranda, C. A. Reitmeier, L. A. Wilson, W. R. Fehr, and J. M. Narvel, "Sensory characteristics of soymilk and tofu made from lipoxygenase-free and normal soybeans," Journal of Food Science, vol. 63, no. 6, pp. 1084-1087, 1998.

[7] D. R. Iassonova, L. A. Johnson, E. G. Hammond, and S. E. Beattie, "Evidence of an enzymatic source of off flavors in 'lipoxygenase- null' soybeans," Journal of the American Oil Chemists' Society, vol. 86, no. 1, pp. 59-64, 2009.

[8] A. V. Torres-Penaranda and C. A. Reitmeier, "Sensory descriptive analysis of soymilk," Journal of Food Science, vol. 66, no. 2, pp. 352-356, 2001.

[9] M. Oozeki, T. Nagamine, T. M. Ikeda et al., "Genetic variation in lipoxygenase activity among Japanese malting barley cultivars and identification of a new lipoxygenase-1 deficient mutant," Breeding Research, vol. 9, no. 2, pp. 55-61, 2007.

[10] H. Ye, S. Harasymow, X.-Q. Zhang et al., "Sequence variation and haplotypes of lipoxygenase gene LOX-1 in the Australian barley varieties," BMC Genetics, vol. 15, article 36, 2014.

[11] Y. Suzuki, I. Kazuo, C. Li, I. Honda, Y. Iwai, and U. Matsukura, "Volatile components in stored rice [Oryza sativa (L.)] of varieties with and without lipoxygenase-3 in seeds," Journal of Agricultural and Food Chemistry, vol. 47, no. 3, pp. 1119-1124, 1999.

[12] I. Feussner and C. Wasternack, "The lipoxygenase pathway," Annual Review of Plant Biology, vol. 53, pp. 275-297, 2002.

[13] K. Mizuno, T. Iida, A. Takano, M. Yokoyama, and T. Fujimura, "A new 9-lipoxygenase cDNA from developing rice seeds," Plant and Cell Physiology, vol. 44, no. 11, pp. 1168-1175, 2003.

[14] S. Ida, Y. Masaki, and Y. Morita, "The isolation of multiple forms and product specificity of rice lipoxygenase," Agricultural and Biological Chemistry, vol. 47, no. 3, pp. 637-641, 2014.

[15] H. Ohta, S. Ida, B. Mikami, and Y. Morita, "Purification and characterization of rice lipoxygenase component 3 from rice," Agricultural and Biological Chemistry, vol. 50, pp. 3165-3171, 1986.

[16] H. Ohta, Y. Shirano, K. Tanaka, Y. Morita, and D. Shibata, "cDNA cloning of rice lipoxygenase L-2 and characterization using an active enzyme expressed from the cDNA in Escherichia coli," European Journal of Biochemistry, vol. 206, no. 2, pp. 331336, 1992.

[17] Y.-L. Peng, Y. Shirano, H. Ohta, T. Hibino, K. Tanaka, and D. Shibata, "A novel lipoxygenase from rice: Primary structure and specific expression upon incompatible infection with rice blast fungus," Journal of Biological Chemistry, vol. 269, no. 5, pp. 37553761, 1994.

[18] Z. He, Study on improving the storable character of rice grains by using genetic engineering [Ph.D. dissertation], Zhejiang University, 2004.

[19] Y. Suzuki and U. Matsukura, "Lipoxygenase activity in maturing and germinating rice seeds with and without lipoxygenase- 3 in mature seeds," Plant Science, vol. 125, no. 2, pp. 119-126, 1997.

[20] G. Tang, G. Galili, and X. Zhuang, "RNAi and microRNA: breakthrough technologies for the improvement of plant nutritional value and metabolic engineering," Metabolomics, vol. 3, no. 3, pp. 357-369, 2007.

[21] D. Miki and K. Shimamoto, "Simple RNAi vectors for stable and transient suppression of gene function in rice," Plant and Cell Physiology, vol. 45, no. 4, pp. 490-495, 2004.

[22] S. Y. Dityatkin, K. V. Lisovskaya, N. N. Panzhava, and B. N. Iliashenko, "Frozen-thawed bacteria as recipients of isolated coliphage DNA," Biochimica et Biophysica Acta (BBA)-Nucleic Acids and Protein Synthesis, vol. 281, no. 3, pp. 319-323, 1972.

[23] Y. Hiei, T. Komari, and T. Kubo, "Transformation of rice mediated by Agrobacterium tumefaciens," Plant Molecular Biology, vol. 35, no. 1-2, pp. 205-218, 1997.

[24] S. Toki, "Rapid and efficient Agrobacterium-mediated transformation in rice," Plant Molecular Biology Reporter, vol. 15, no. 1, pp. 16-21, 1997.

[25] M. RoyChowdhury, J. A. Dabul, and P. G. C. Hubstenberger, "Biotechnology advances for Arkansas rice varieties (Oryza sativa L.)," Arkansas Academy of Science, vol. 60, pp. 113-118, 2007.

[26] P. Chomczynski and N. Sacchi, "Single-step method of RNA isolation by acid guanidinium thiocyanate-phenol-chloroform extraction," Analytical Biochemistry, vol. 162, no. 1, pp. 156-159, 1987.

[27] M. W. Pfaffl, "A new mathematical model for relative quantificationin real-time RT-PCR," Nucleic Acids Research, vol. 29, pp. 2002-2007, 2001.

[28] M. W. Pfaffl, G. W. Horgan, and L. Dempfle, "Relative Expression Software Tool (REST) for group-wise comparison and 
statistical analysis of relative expression results in real-time PCR," Nucleic Acids Research, vol. 30, no. 9, article e36, 2002.

[29] R. Bryant, A. Proctor, M. Hawkridge et al., "Genetic variation and association mapping of silica concentration in rice hulls using a germplasm collection," Genetica, vol. 139, no. 11-12, pp. 1383-1398, 2011.

[30] C. C. Grimm, C. Bergman, J. T. Delgado, and R. Bryant, "Screening for 2-acetyl-1-pyrroline in the headspace of rice using SPME/GC-MS," Journal of Agricultural and Food Chemistry, vol. 49, no. 1, pp. 245-249, 2001.

[31] J. Gil-Humanes, F. Pistón, A. Hernando, J. B. Alvarez, P. R. Shewry, and F. Barro, "Silencing of $\gamma$-gliadins by RNA interference (RNAi) in bread wheat," Journal of Cereal Science, vol. 48, no. 3, pp. 565-568, 2008.

[32] A. Regina, A. Bird, D. Topping et al., "High-amylose wheat generated by RNA interference improves indices of large-bowel health in rats," Proceedings of the National Academy of Sciences of the United States of America, vol. 103, no. 10, pp. 3546-3551, 2006.

[33] D. Yin, S. Deng, K. Zhan, and D. Cui, "High-oleic peanut oils produced by HpRNA-mediated gene silencing of oleate desaturase," Plant Molecular Biology Reporter, vol. 25, no. 3-4, pp. 154-163, 2007.

[34] S. Liu and B. Han, "Differential expression pattern of an acidic 9/13-lipoxygenase in flower opening and senescence and in leaf response to phloem feeders in the tea plant," BMC Plant Biology, vol. 10, article 228, pp. 1471-2229, 2010.

[35] T. K. Park and J. C. Polacco, "Distinct lipoxygenase species appear in the hypocotyl/radicle of germinating Soybean," Plant Physiology, vol. 90, no. 1, pp. 285-290, 1989.

[36] U. Schaffrath, F. Zabbai, and R. Dudler, "Characterization of RCI-1, a chloroplastic rice lipoxygenase whose synthesis is induced by chemical plant resistance activators," European Journal of Biochemistry, vol. 267, no. 19, pp. 5935-5942, 2000.

[37] Z. Kong and D. Zhao, "The inhibiting effect of abscisic acid on fragrance of kam sweet rice," Journal of Food and Nutrition Research, vol. 2, no. 4, pp. 148-154, 2014.

[38] S. S. Marla and V. K. Singh, "LOX genes in blast fungus (Magnaporthe grisea) resistance in rice," Functional and Integrative Genomics, vol. 12, no. 2, pp. 265-275, 2012. 

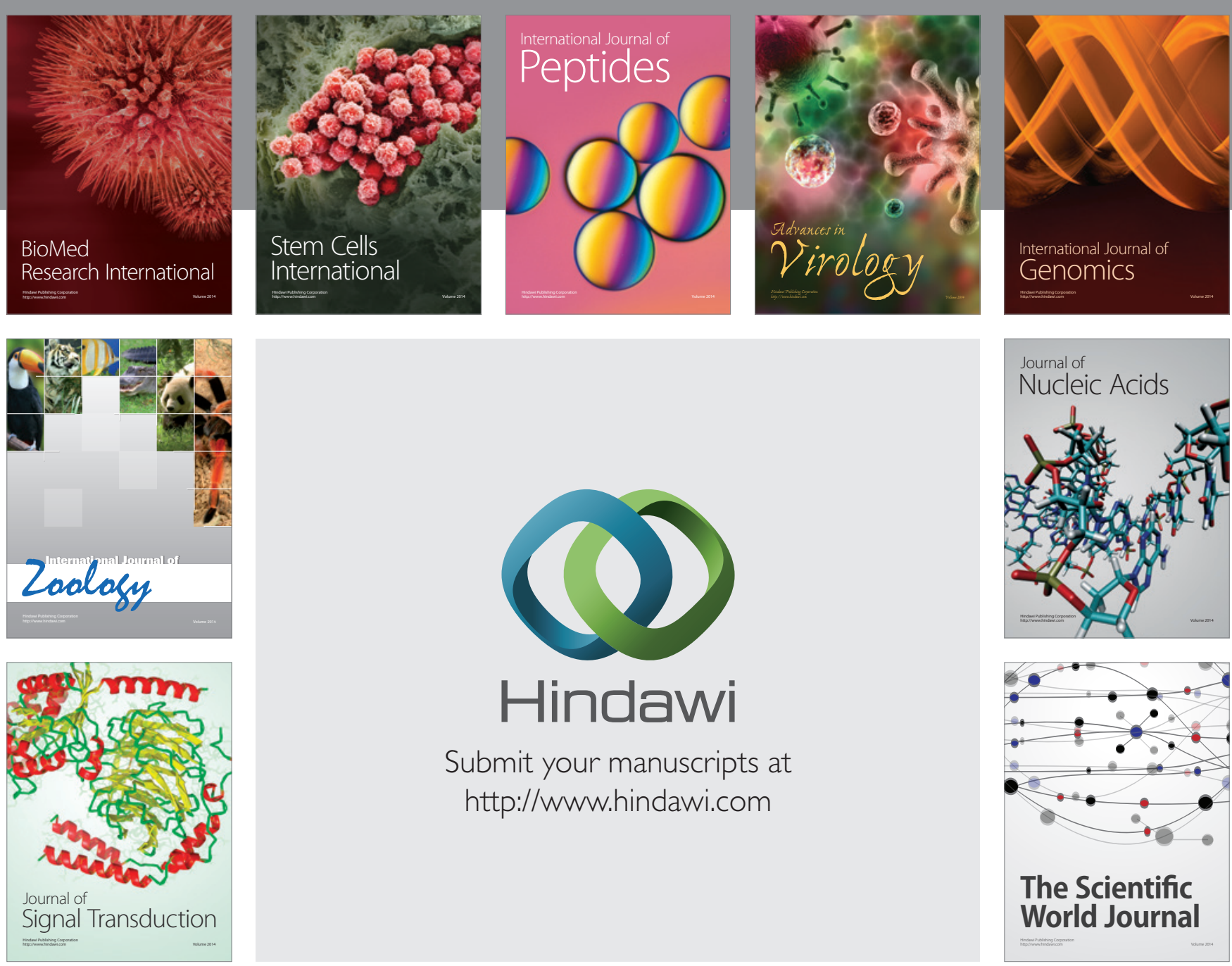

Submit your manuscripts at

http://www.hindawi.com
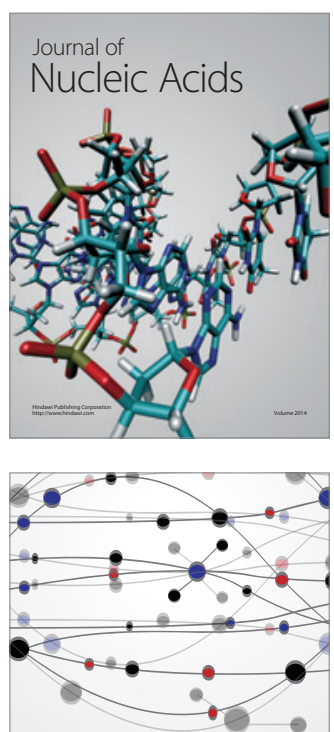

The Scientific World Journal
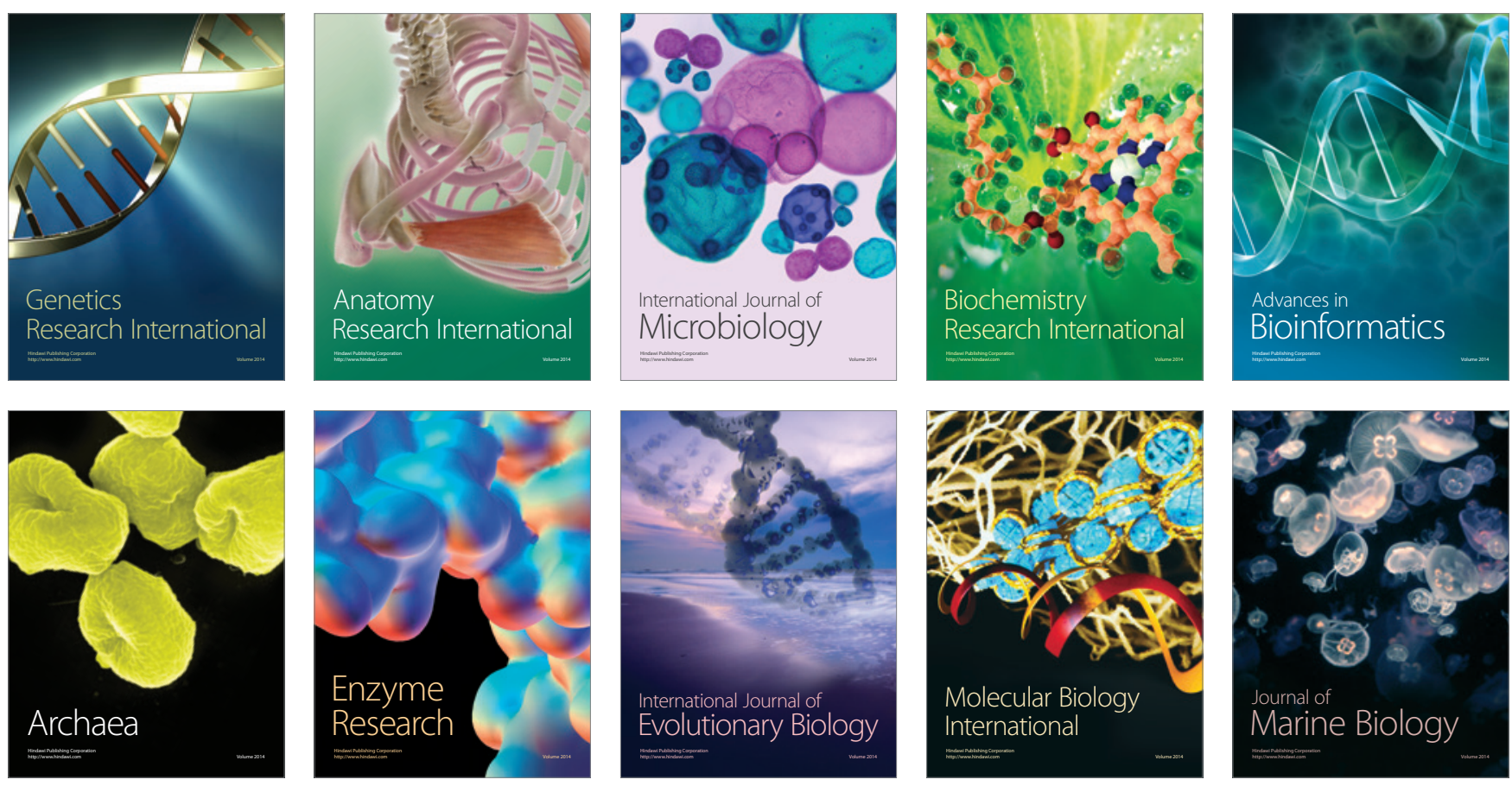